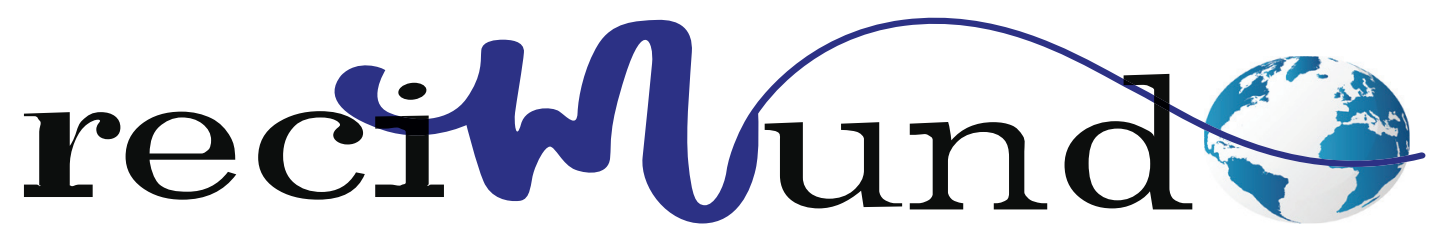

Revista Científica Mundo de la Investigación y el Conocimiento

DOI: 10.26820/recimundo/5.(2).abril.2021.16-25

URL: https://recimundo.com/index.php/es/article/view/1029

EDITORIAL: Saberes del Conocimiento

REVISTA: RECIMUNDO

ISSN: 2588-073X

TIPO DE INVESTIGACIÓN: Artículo de revisión

CÓDIGO UNESCO: 32 Ciencias Médicas

PAGINAS: $16-25$

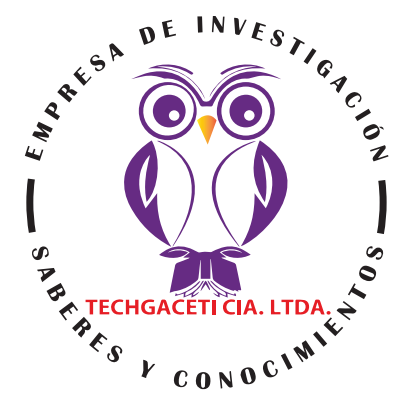

\title{
Utilidad del ecofast en pacientes con traumatismo toráxico ingresados en urgencias
}

Usefulness of echofast in patients with thoracic trauma admitted to the emergency department

Utilidade do ecofast em pacientes com trauma torácico admitidos no departamento de emergência

Luis Adrián Muñoz Andrade1; Rosana Fabregas Almanza2; Jeyson Eddy Roth Chuizaca3;

Andrea Geovanna Sanipatín Criollo ${ }^{4}$

\section{RECIBIDO: 15/01/2021 ACEPTADO: 20/03/2021 PUBLICADO: 01/04/2021}

1. Médico Institucional Consejo Nacional Electoral; Quito, Ecuador; munozandradeluis@gmail.com; (iD https://orcid.org/00000002-9682-5896

2. Médico General en Funciones Hospitalarias; rochyfa@hotmail.com; (iD https://orcid.org/0000-0001-9756-9102

3. Médico General Clínica Villaflora; Quito, Ecuador; jroth@udlanet.ec; (D) https://orcid.org/0000-0001-8570-6245

4. Médico General de Libre Ejercicio Profesional; annsanipatin@gmail.com; (DD https://orcid.org/0000-0003-3928-340X

CORRESPONDENCIA

Luis Adrián Muñoz Andrade

munozandradeluis@gmail.com

Quito, Ecuador 


\section{RESUMEN}

La evaluación enfocada con ecografía en trauma (FAST) se ha utilizado y estudiado ampliamente en trauma contundente y penetrante durante las últimas 3 décadas. Antes de FAST, los procedimientos invasivos como el lavado peritoneal de diagnóstico y la laparotomía exploratoria se utilizaban comúnmente para diagnosticar lesiones intraabdominales. En la actualidad, el examen FAST se ha convertido en un estudio más completo del abdomen, el corazón, el tórax, la vena cava inferior, entre otras, existiendo muchas variaciones en la técnica, los protocolos y la interpretación. Las estrategias de manejo de traumatismos como laparotomía, endoscopia, angiografía por tomografía computarizada, intervención angiográfica, imágenes seriadas y observación clínica también han cambiado a lo largo de los años. Esta técnica, en ocasiones, ha logrado reemplazar la tomografía computarizada y el diagnóstico de lavado peritoneal, sin producir retrasos en el procedimiento quirúrgico. En tal sentido, la relación entre la información clínica del paciente y los resultados del examen deben estar guiados a la orientación de los enfoques terapéuticos en escenarios de difícil acceso como unidades de cuidados intensivos en zonas de guerra, lugares rurales o distantes, donde otros métodos de imagenología no están disponibles. Esta revisión discutirá la evolución del examen FAST a su estado actual y evaluará su papel en evolución en el manejo agudo del paciente traumatizado.

Palabras clave: Ecografía, trauma; cuidados intensivos, sistemas en el punto de atención.

\section{ABSTRACT}

Focused trauma ultrasound assessment (FAST) has been used and studied extensively in blunt and penetrating trauma over the past 3 decades. Before FAST, invasive procedures such as diagnostic peritoneal lavage and exploratory laparotomy were commonly used to diagnose intra-abdominal injuries. Today, the FAST examination has become a more comprehensive study of the abdomen, heart, thorax, inferior vena cava, among others, with many variations in technique, protocols, and interpretation. Trauma management strategies such as laparotomy, endoscopy, computed tomography angiography, angiographic intervention, serial imaging, and clinical observation have also changed over the years. This technique, on occasion, has succeeded in replacing computed tomography and peritoneal lavage diagnosis, without causing delays in the surgical procedure. In this sense, the relationship between the clinical information of the patient and the results of the examination should be guided to the orientation of therapeutic approaches in settings of difficult access such as intensive care units in war zones, rural or distant places, where other methods imaging are not available. This review will discuss the evolution of the FAST exam to its current state and assess its evolving role in the acute management of the trauma patient.

Keywords: Ultrasound, trauma; intensive care, systems at the point of care.

\section{RESUMO}

A avaliação ultra-sonográfica focalizada do trauma (FAST) tem sido usada e estudada extensivamente em traumatismos rombos e penetrantes nas últimas 3 décadas. Antes do FAST, procedimentos invasivos, tais como lavagem peritoneal diagnóstica e laparotomia exploratória eram comumente usados para diagnosticar lesões intra-abdominais. Hoje, o exame FAST tornou-se um estudo mais abrangente do abdômen, coração, tórax, veia cava inferior, entre outros, com muitas variações na técnica, protocolos e interpretação. As estratégias de manejo de trauma como laparotomia, endoscopia, angiografia computadorizada, intervenção angiográfica, imagens seriadas e observação clínica também mudaram ao longo dos anos. Esta técnica, em algumas ocasiões, conseguiu substituir a tomografia computadorizada e o diagnóstico de lavagem peritoneal, sem causar atrasos no procedimento cirúrgico. Neste sentido, a relação entre a informação clínica do paciente e os resultados do exame deve ser orientada para a orientação de abordagens terapêuticas em ambientes de difícil acesso, tais como unidades de terapia intensiva em zonas de guerra, rurais ou distantes, onde outros métodos de imagem não estão disponíveis. Esta revisão discutirá a evolução do exame RÁPIDO ao seu estado atual e avaliará seu papel evolutivo no manejo agudo do paciente traumatizado.

Palavras-chave: Ultra-som, trauma; cuidados intensivos, sistemas no ponto de atendimento. 


\section{Introducción}

Las lesiones traumáticas siguen siendo la principal causa de muerte entre las personas de 1 a 44 años. "En 2013, hubo 27 millones de pacientes tratados en los departamentos de emergencia, con 3 millones hospitalizados por sus lesiones solamente en los Estados Unidos" (National Center for Injury Prevention and Control, 2015). Una proporción sustancial de estos pacientes tiene lesiones por traumatismo abdominal cerrado y / o torácico.

La llegada de la evaluación enfocada con ecografía en trauma (FAST) hace 3 décadas permitió a los médicos detectar rápidamente lesiones al lado de la cama de los pacientes, especialmente aquellos pacientes demasiado inestables hemodinámicamente para el transporte a la sala de tomografía computarizada (TC).

La identificación de líquido libre dentro de la cavidad peritoneal, el pericardio y los espacios pleurales se puede lograr inmediatamente al llegar el paciente al hospital. Otras aplicaciones de FAST incluyen la detección de lesiones de órganos sólidos, neumotórax, fracturas, exámenes seriados, así como su uso en el transporte prehospitalario y en entornos de múltiples víctimas como herramienta de clasificación. (Catán, Villao, \& Astudillo, 2011)

Sin embargo, existen opiniones encontradas entre los radiólogos a adoptar el uso de la ecografía en traumatismos, ya que existe una mayor dependencia de la TC. "Mucho de esto se debe al hecho de que el uso de FAST ha migrado a los primeros respondedores e incluye el uso de FAST en el campo o durante el transporte de pacientes" (Kool \& Blickman, 2017). El FAST también se usa típicamente como examen de imagen inicial del paciente al llegar al departamento de emergencias. Desde la descripción original del uso de la ecografía en el paciente traumatizado, se encuentran varias aplica- ciones nuevas del uso de la ecografía en estos pacientes.

El ultrasonido se utilizó por primera vez para el examen de pacientes traumatizados en la década de 1970 en Europa. No se adoptó ampliamente en América del Norte hasta la década de 1990, tiempo durante el cual el acrónimo FAST se definió como "ecografía abdominal enfocada para traumatismos" (Rozycki, Ochsner, \& Schmidt, 2005).

A medida que FAST evolucionó hacia un examen más completo, el acrónimo se cambió a "evaluación enfocada con ecografía para traumatismos". Desde entonces, "FAST se ha convertido en la modalidad de detección inicial común en la mayoría de los centros de trauma en todo el mundo, y está incluido en el programa Advanced Trauma Life Support para evaluaración del paciente traumatizado hipotensivo" (Kool \& Blickman, 2017). Un aspecto único de FAST es que lo utilizan habitualmente radiólogos, médicos de urgencias y cirujanos con formación y experiencia variables.

\section{Metodología}

Esta investigación está enfocada en el estudio de la utilidad del ecofast en pacientes con traumatismo toráxico ingresados en urgencias con la finalidad de dar a conocer los beneficios así como también, los contra que este tipo de procedimientos presenta ante una situación clínica.

La revisión se ha centrado en textos, documentos y artículos científicos publicados disponibles en la web, considerando que aquella herencia de la globalización permite acceder a mayor y mejor información a través de las herramientas tecnológicas. El motor de búsqueda ha sido herramientas académicas de la web que direccionan específicamente a archivos con validez y reconocimiento científico, descartando toda información no confirmada o sin las respectivas referencias bibliográficas. 


\section{Resultados}

\section{Interpretación de la técnica FAST}

La selección de la sonda en la evaluación del paciente traumatizado depende de cuál sea el enfoque principal del examen. Una sonda sectorial $(3-5 \mathrm{MHz})$ se utiliza mejor como sonda multipropósito. "Es apropiado para examinar órganos sólidos y determinar la presencia de líquido libre en el abdomen o la pelvis. Se puede utilizar un escáner de sector para examinar el corazón en busca de derrame pericárdico o hemorragia" (Carter, Falco, \& Chopko, 2015). Un escáner de sector también es útil para escanear entre las costillas en busca de neumotórax.

Se puede usar un transductor de matriz curva en el abdomen para una mejor resolución, pero no es ideal para obtener imágenes del corazón o los pulmones, especialmente cuando se escanea en los espacios intercostales. Los transductores de matriz lineal no son ideales debido a su huella más grande en el abdomen y el pecho y, a menudo, son de mayor frecuencia con una penetración de profundidad limitada. (Carter, Falco, \& Chopko, 2015)

De acuerdo con, lanniello \& Di Giacomo, (2014) la exploración FAST original incluía "vistas de a) el cuadrante superior derecho, incluía el área perihepática y el receso hepatorrenal o bolsa de Morison, b) cuadrante superior izquierdo, abarca vista periesplénica, c) proyección suprapúbica (bolsa de Douglas), y posteriormente d) proyección pericárdica subxifoidea" (Fig. 1).

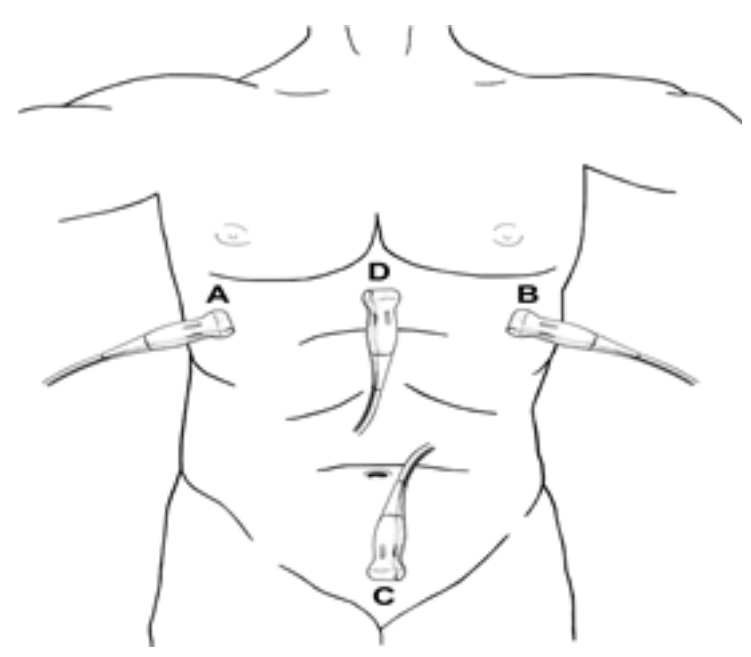

Figura 1. Los cuatro vistas para la exploración FAST original: $\mathrm{A}$ = cuadrante superior derecho, $\mathrm{B}=$ cuadrante superior izquierdo, $C$ = vista suprapúbica, $D=$ vista subxifoidea del corazón

Fuente: (lanniello \& Di Giacomo, 2014)

El sitio inicial preferido para la detección de líquido libre con FAST es la vista del cuadrante superior derecho, escaneada mediante un sector de frecuencia inferior $(3,5-5 \mathrm{MHz})$ o un transductor de matriz curva. "Un transductor de sector con campo lejano optimizado es ideal para una mejor penetración al examinar la fosa hepatorrenal o la pelvis profunda" (Rozycki, Ochsner, \& Schmidt, 2005). "También se puede optimizar un transductor de matriz curva para una penetración profunda. Sin embargo, los transductores de matriz lineal rara vez se utilizan en el abdomen" (Ianniello \& Di Giacomo, 2014)

El hígado sirve como una ventana acústica conveniente para interrogar el espacio hepatorrenal y el parénquima hepático. "El hemoperitoneo suele aparecer anecoico o hipoecoico en comparación con órganos sólidos adyacentes. La hemorragia prolongada puede organizarse y volverse ecogénica. Para vista del cuadrante superior izquierdo, el bazo se enfoca para examinar la fosa esplenorrenal y el área peri esplénica" (Kool \& Blickman, 2017). 
La exploración cefálica permite la visualización del espacio pleural izquierdo. Al mover la sonda caudalmente, se visualizan el polo inferior del riñón izquierdo y el canal paracólico. El área peri esplénica "puede escanearse de manera inadecuada debido al difícil acceso físico. Girar al paciente hacia el lado derecho es útil para evaluar esta área, ya que pueden acumularse pequeñas cantidades de líquido libre en la parte superior del bazo" (Volpicelli, 2011).

La vista suprapúbica permite evaluar el espacio más dependiente de la cavidad peritoneal.

El transductor se coloca por encima de la sínfisis púbica en un plano sagital y se desplaza de lado a lado, luego se gira transversalmente y se repite. La posición de Trendelenburg inversa puede mejorar la detección de líquido libre en la pelvis. En pacientes femeninas en edad reproductiva, pequeñas cantidades de líquido libre de hasta $50 \mathrm{ml}$ en la bolsa de Douglas se consideran fisiológicas y cantidades superiores a $50 \mathrm{ml}$ deben considerarse patológicas en el contexto del trauma (Ormsby, Geng, McGahan, \& Richards, 2015).

Por lo tanto, suponiendo que no haya una lesión u otra condición patológica presente, no se debe encontrar líquido libre en el espacio recto vesicular en los hombres. Solo se deben encontrar pequeñas cantidades de líquido en el espacio recto-uterino en mujeres en edad fértil. "La detección de líquido libre en la pelvis se ve favorecida por la presencia de una vejiga llena de líquido. Cuando hay líquido libre, lo más frecuente es que se localice posterior o superior a la vejiga y / o al útero" (Ormsby, Geng, McGahan, \& Richards, 2015).

El líquido libre en la pelvis se puede perder cuando se coloca un catéter de Foley para vaciar la vejiga, ya que la ventana acústica para examinar la pelvis está comprometida, lo que permite la detección de solo gran- des cantidades de líquido pélvico. El examen óptimo para la detección de cantidades más pequeñas de líquido libre pélvico requiere una vejiga más distendida, permitiendo la detección de solo grandes cantidades de líquido pélvico.

Existen limitaciones para el examen FAST independientemente del protocolo utilizado. "Para la exploración abdominal, la detección de lesiones contusas mesentéricas, intestinales, diafragmáticas y retroperitoneales puede ser difícil, así como una lesión penetrante aislada del peritoneo" (Manson \& Hafez, 2011).

Las exploraciones falsas positivas pueden resultar de la detección de ascitis, dializado peritoneal, salida de la derivación ventriculoperitoneal, hiperestimulación ovárica y rotura de un quiste ovárico. La reanimación masiva de volumen intravascular puede resultar en un examen FAST falso positivo de trasudación de líquido intravascular a intraperitoneal (Slutzman, Arvold, Rempell, Stone, \& Kimberly, 2014).

Aunque se supone que el líquido libre detectado con FAST en pacientes traumatizados es hemoperitoneo, también puede representar contenido de orina, bilis e intestino relacionado con la lesión. Los gases intestinales, el enfisema subcutáneo y la obesidad representan obstáculos comunes para la visualización ecográfica completa.

Los pacientes con presentación tardía después de un traumatismo pueden tener coágulos que contienen hemoperitoneo que pueden tener una ecogenicidad mixta y pasar desapercibidos. La grasa perirrenal, que ensancha la interfaz hepatorrenal y esplenorrenal, puede malinterpretarse como líquido libre o hematoma subcapsular, también conocido como el signo de la "línea doble" (Sierzenski, Schofer, Bauman, \& Nomura, 2011). 
El volumen de líquido libre necesario para permitir la detección con FAST representa una limitación de FAST. Los autores Hernández \& Gutiérrez, (2015) expresan que "el volumen de líquido libre mínimo detectable medio durante el examen FAST en 100 pacientes sometidos a DPL fue de $619 \mathrm{ml}$ en la bolsa de Morison". La posición de Trendelenburg puede mejorar la visualización del líquido libre en la interfaz esplenorrenal y hepatorrenal. Sin embargo, Carter, Falco, \& Chopko, (2015) "demostraron que FAST realizado en la posición de Trendelenburg permitió la detección de cantidades más pequeñas de líquido libre hepatorrenal que en decúbito supino (mediana, $400 \mathrm{ml}$ frente a $700 \mathrm{ml}) "$.

En otro estudio de DPL, Von Kuenssberg Jehle, Stiller, \& Wagner, (2013) "determinaron que se requerían volúmenes aún más pequeños para la detección en las vistas pélvicas de FAST, con una mediana de volumen mínimo de líquido de 100 ml". Sin embargo, otros estudios han demostrado una capacidad limitada de detección de pequeñas cantidades de líquido pélvico libre con el abordaje transabdominal después de la descompresión de la vejiga con cateterismo de Foley.

\section{Protocolos más nuevos}

A mediados de la década de 2000, la adición de la evaluación estadounidense del tórax para detectar neumotórax al examen FAST tradicional resultó en FAST extendido (eFAST). Hay varios otros protocolos desarrollados para la evaluación del shock, la dificultad respiratoria y el paro cardíaco, algunos de los cuales incluyen ecocardiografía. Otros protocolos para la evaluación de la disnea incluyen BLUE (ecografía pulmonar junto a la cama en caso de emergencia) y RADIUS (evaluación rápida de la disnea con ecografía). El protocolo BLUE incluye solo ecografía pulmonar para la detección de neumotórax, así como edema pulmonar, consolidación y derrame. El protocolo RA-
DIUS es similar pero incluye evaluación cardíaca y de la vena cava inferior (VCI) (Manson \& Hafez, 2011).

No es posible una revisión de todos los protocolos, pero algunos merecen una revisión más a fondo. Los autores Manson \& Hafez, (2011) "del protocolo RUSH (un acrónimo de ecografía rápida para shock e hipotensión) simplificaron su conceptualización como un examen de (a) bomba, (b) tanque y (c) tuberías". La evaluación de la "bomba" incluye los ejes paraesternal largo y corto del corazón, además de las proyecciones subxifoidea y apical. La evaluación de "tanque" implica el interrogatorio de la $\mathrm{VCl}$, un examen RÁPIDO del abdomen que incluye vistas pleurales y una ecografía del pulmón.

La porción de "conductos" de RUSH implica la exploración de la aorta supraesternal, paraesternal, epigástrica y supraumbilical, con exploraciones adicionales de las venas femoral y poplítea en busca de trombosis venosa profunda. "El examen RUSH no está dirigido específicamente a pacientes traumatizados, por lo tanto, la parte de "tuberías" del protocolo generalmente no se realiza en el contexto de un traumatismo agudo" (Ghane \& Gharib, 2015). Hasta donde se sabe, actualmente no hay estudios publicados que evalúen específicamente el examen RUSH exclusivamente para pacientes con traumatismos hipotensos.

Los autores Ghane \& Gharib, (2015) informaron "una sensibilidad del 100\% (16 de 16) para RUSH en el diagnóstico de choque hipovolémico en 16 pacientes, cinco de los cuales tenían lesiones de órganos sólidos secundarios a un traumatismo abdominal cerrado". Los pacientes restantes en su estudio fueron diagnosticados con shock por condiciones médicas agudas, cinco de los cuales tenían lesiones de órganos sólidos secundarios a un traumatismo abdominal cerrado. 
La cantidad de protocolos diferentes para la evaluación del paciente grave o enfermo es una fuente de confusión, especialmente porque se desarrollan aún más protocolos con siglas y abreviaturas creativas. Sería útil establecer un protocolo de examen estandarizado por consenso y basado en grandes estudios prospectivos y / o metanálisis. "De estos protocolos, el examen eFAST, que incluye la evaluación de neumotórax, y partes del examen RUSH, que incluye una breve vista subcostal del corazón y la evaluación de la VCI, parecen más prácticos y eficientes en el tiempo" (Manson \& Hafez, 2011) (Fig2).

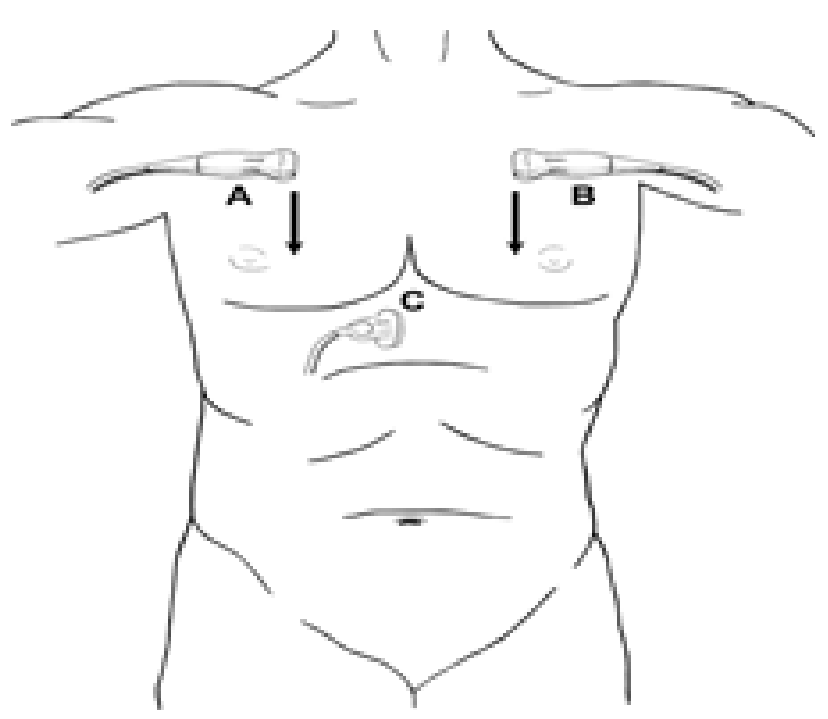

Figura 2. Vistas adicionales que pueden ser útiles en el paciente traumatizado: $A=$ vista parasagital derecha del pulmón para neumotórax, $\mathrm{B}=$ vista parasagital izquierda del pulmón para neumotórax, $\mathrm{C}=$ vista longitudinal de la VCl.

Fuente: (Manson \& Hafez, 2011)

\section{Corazón}

Las imágenes subxifoides del corazón se obtienen colocando el transductor en la parte superior del abdomen y apuntando hacia arriba, hacia el hombro izquierdo. El líquido que rodea el corazón se ve como un espacio anecoico que rodea el miocardio. El hígado actúa como una ventana acústica. Si hay dificultades para obtener la proyección subxifoidea, se pueden intentar abordajes paraesternal, apical de cuatro cámaras y subcostal.

Si se detecta una cantidad sustancial de hemopericardio, es probable que se produzca un taponamiento cardíaco si hay colapso diastólico de la aurícula y / o el ventrículo derechos. El líquido en el espacio pericárdico posterior puede ser difícil de distinguir del líquido en la cavidad pleural posteromedial. Se puede hacer una distinción visualizando la aorta torácica descendente, ya que el líquido pericárdico está presente anterior a la aorta mientras que el líquido pleural es posterior (Ghane \& Gharib, 2015).

Los resultados falsos positivos para el hemopericardio incluyen quiste pericárdico, almohadilla grasa y derrame preexistente. El área pericárdica subxifoidea puede escanearse de manera inadecuada debido a una ventana acústica subóptima.

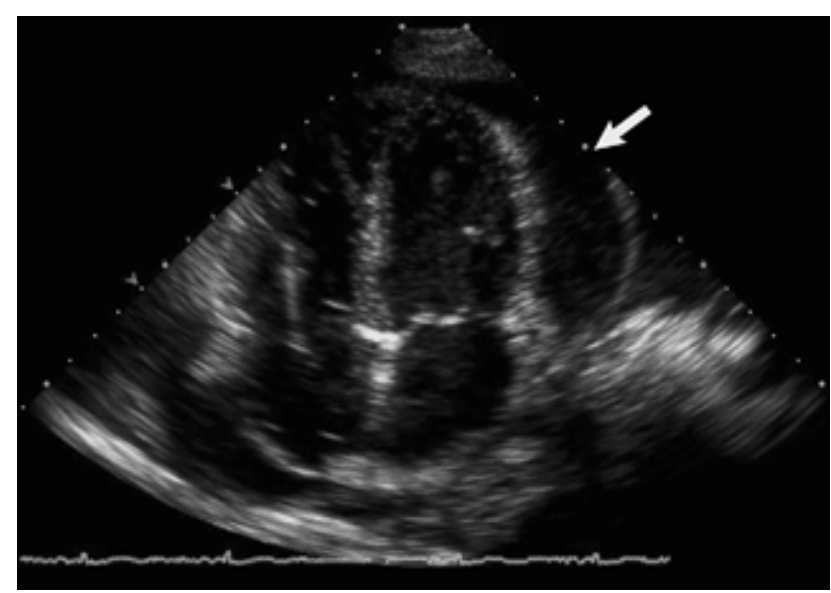

Figura 3. Derrame pericárdico: la vista de cuatro cámaras del corazón muestra un derrame pericárdico de tamaño moderado (flecha).

Fuente: (Ghane \& Gharib, 2015)

\section{Hemotórax o derrame pleural}

En este momento, se puede explorar el espacio pleural derecho en busca de líquido libre, así como la interfaz entre la cúpula del 
hígado y el diafragma. Esta interfaz aparece como una línea curvilínea ecogénica, y en la parte superior se pueden ver ecos similares al parénquima hepático. "Este artefacto de imagen especular sugiere la ausencia de líquido pleural. El pulmón normal puede distorsionar intermitentemente esta interfaz durante la inspiración, lo que se conoce como el "signo de la cortina" (Ghane \& Gharib, 2015).

El líquido pleural puede ser anecoico o tener ecogenicidad mixta según su composición (p. Ej., Hemorragia, exudado, trasudado, empiema). También se puede ver el pulmón atelectásico con esta vista. La posición de Trendelenburg vertical o invertida puede mejorar la detección de líquido pleural.

\section{Neumotórax}

Como eFAST es un protocolo relativamente nuevo, hay menos estudios que evalúen su precisión en la detección de neumotórax. El diagnóstico de neumotórax "de tamaño pequeño a moderado con examen físico y radiografía de tórax en decúbito supino es un desafío, y estas lesiones ocultas pueden pasarse por alto hasta en el 76\% (81 de $107)$ de los pacientes con traumatismos cerrados" (National Center for Injury Prevention and Control, 2015).

En los estudios que utilizan la TC como estándar de referencia, la sensibilidad de eFAST es mejor que la de la radiografía de tórax en decúbito supino. Los autores Kirkpatrick, Sirois, \& Laupland, (2004) "realizaron un estudio prospectivo ciego de 225 pacientes traumatizados con eFAST e informaron una sensibilidad del 48,8\% (21 de 43) para la ecografía de tórax frente al 20,9\% (nueve de 43) para la radiografía de tórax".

Adicionalmente lanniello \& Di Giacomo, (2014) "investigaron a 368 pacientes traumatizados inestables con eFAST e informaron una sensibilidad del 77\% (67 de 87) para la detección de neumotórax"

Para la detección de neumotórax, una frecuencia alta(>5 MHz) se prefiere una sonda de transductor lineal, pero también se pueden usar transductores de sector de frecuencia más baja e incluso un transductor curvo. El transductor se coloca "en el segundo o tercer espacio intercostal en la línea medio clavicular en orientación sagital, luego se mueve hacia abajo. La sonda también se puede colocar de forma oblicua entre las costillas para obtener una vista más amplia del pulmón" (Catán, Villao, \& Astudillo, 2011). La sonda debe colocarse en diferentes posiciones en la parte anterior del tórax y compararse con el lado opuesto para verificar si hay neumotórax.

El enfisema subcutáneo puede obstruir los intentos de ecografía de la cavidad pleural subyacente y se asocia con frecuencia con neumotórax. La gravedad de la enfermedad puede "ser un factor, ya que el valor predictivo positivo de la ausencia de deslizamiento pulmonar para detectar neumotórax es del $87 \%$ en la población general, del $56 \%$ en los enfermos críticos y del $27 \%$ en los pacientes con insuficiencia respiratoria" (Lichtenstein \& Menu, 2005).

Un neumotórax solo se puede detectar directamente debajo de la sonda, y es posible que se pasen por alto neumotórax localizado más pequeños. Los neumotórax apicales son más difíciles de detectar porque hay un menor grado de movimiento pulmonar en comparación con el tórax inferior.

\section{Conclusión}

La ecografía ha revolucionado la atención de lesiones traumáticas. Numerosos estudios, aunque en su mayoría observacionales, han demostrado que el protocolo eFAST es un complemento clínicamente significativo en la evaluación y el tratamiento de pacientes traumatizados. El eFAST se recomienda como el estándar de atención

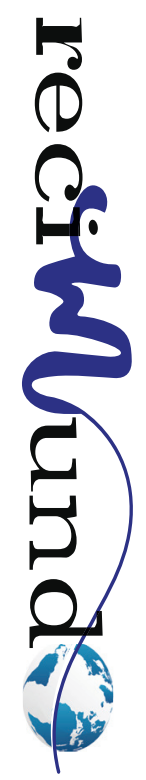


en los protocolos de reanimación de trauma. Se ha demostrado que reduce el tiempo de intervención quirúrgica; duración de la estancia del paciente; costo; y las tasas de complicaciones, TC y DPL realizadas. Sin embargo, al igual que con cualquier modalidad de diagnóstico por imágenes, se reconocen y comprenden sus limitaciones.

Es evidente que, el tratamiento de los pacientes traumatizados suele estar a cargo de un equipo interprofesional que incluye enfermeras traumatológicas. A pesar de los cuestionamientos y limitaciones, el FAST es útil para los pacientes que presentan trauma. Sin embargo, los médicos deben saber que la adquisición e interpretación de imágenes ecográficas en el punto de atención están limitadas por la experiencia del proveedor; habitus corporal del paciente; y la presencia de gases intestinales, neumoperitoneo o neumomediastino. En estas situaciones, se justifican los exámenes eFAST en serie y las imágenes avanzadas en función del estado hemodinámico del paciente. Adicionalmente, de ser necesario, se debe consultar a un radiólogo si uno no puede interpretar las imágenes.

\section{Bibliografía}

Bolívar, J. (2015). Investigación Documental. México. Pax.

Carter, J., Falco, M., \& Chopko, M. (2015). Do we really rely on fast for decision-making in the management of blunt abdominal trauma? Injury , 817-821.

Castro, J. (2016). Técnicas Documentales. México. Limusa.

Catán, F., Villao, D., \& Astudillo, C. (2011). Ecografía fast en la evaluación de pacientes traumatizados. Revista Médica Clínica Las Condes , 2 (5), 633639.

Davila, A. (2015). Diccionario de Términos Científicos. . Caracas: Editorial Oasis.

Ghane, M., \& Gharib, M. (2015). Accuracy of rapid ultrasound in shock (RUSH) exam for diagnosis of shock in critically ill patients. Trauma Mon, 200-95.

Hernández, N., \& Gutiérrez, M. (2015). US FAST. REVISTA MEDICA DE COSTA RICA Y CENTROAME-
RICA , 614, 65-68.

Ianniello, S., \& Di Giacomo, V. (2014). First-line sonographic diagnosis of pneu- mothorax in major trauma: accuracy of e- FAST and comparison with multidetector computed tomography. Radiol Med , 674-680.

Kirkpatrick, A., Sirois, M., \& Laupland, K. (2004). Hand-held thoracic sonography for detecting post-traumatic pneumothoraces: the Extended Focused Assessment with Sonography for Trauma (EFAST). J Trauma , 288-295.

Kool, D., \& Blickman, J. (2017). Advanced Trauma Life Support. ABCDE from a radiological point of view. Emerg Radiol , 135-141.

Lichtenstein, D., \& Menu, Y. (2005). A bedside ultrasound sign ruling out pneumothorax in the critically ill. Lung sliding. Chest, 1345-1348.

Manson, W., \& Hafez, N. (2011). The rapid assessment of dyspnea with ultrasound: RADiUS. Ultrasound Clin , 261-276.

National Center for Injury Prevention and Control. (2015, December 01). Centers for Disease Control and Prevention. Retrieved from http://www.cdc. gov/injury/wisqars/overview/key_data.html

Ormsby, E., Geng, J., McGahan, J., \& Richards, J. (2015). Pelvic free fluid: clinical importance for reproductive age women with blunt abdominal trauma. Ultrasound Obstet Gynecol , 271-278.

Rozycki, G., Ochsner, M., \& Schmidt, J. (2005). A prospective study of surgeon-performed ultrasound as the primary adjuvant modality for injured patient assessment. J Trauma , 498-500.

Sierzenski, P., Schofer, J., Bauman, M., \& Nomura, J. (2011). The double-line sign: a false positive finding on the Focused Assessment with Sonography for Trauma (FAST) exam- ination. J Emerg Med, 188-189.

Slutzman, J., Arvold, L., Rempell, J., Stone, M., \& Kimberly, H. (2014). Positive FAST without hemoperitoneum due to fluid re- suscitation in blunt trauma. J Emerg Med , 427-429.

Volpicelli, G. (2011). Sonographic diagnosis of pneumothorax. Intensive Care Med , 224-232.

Von Kuenssberg Jehle, D., Stiller, G., \& Wagner, D. (2013). Sensitivity in detecting free intraperito- neal fluid with the pelvic views of the FAST exam. Am J Emerg Med , 476- 478. 


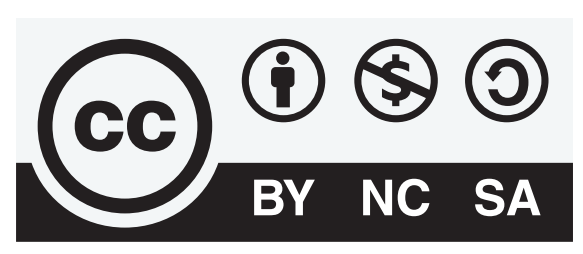

CREATIVE COMMONS RECONOCIMIENTO-NOCOMERCIAL-COMPARTIRIGUAL 4.0 .

\section{CITAR ESTE ARTICULO:}

Muñoz Andrade, L. A., Fabregas Almanza, R., Roth Chuizaca, J. E., \& Sanipatín Criollo, A. G. (2021). Utilidad del ecofast en pacientes con traumatismo toráxico ingresados en urgencias. RECIMUNDO, 5(2), 16-25. https://doi. org/10.26820/recimundo/5.(2).abril.2021.16-25 